\title{
Processing Array Statements and Procedure Interfaces in the PREPARE High Performance Fortran Compiler *
}

\author{
Siegfried Benkner, Peter Brezany and Hans Zima \\ Institute for Software Technology and Parallel Systems, University of Vienna, \\ Brünnerstrasse 72, A-1210 Vienna, Austria
}

\begin{abstract}
Recently, a standard set of extensions for Fortran 90, called High Performance Fortran (HPF), has been developed which would provide a portable interface to a wide variety of parallel architectures. HPF focuses mainly on issues of distributing data across the memories of a distributed memory multiprocessor. This paper proposes techniques for processing $\mathrm{HPF}$ data distribution and data alignment specifications, array statements and procedure interfaces in the HPF compiler which is being developed in the ESPRIT project Prepare.
\end{abstract}

\section{Introduction}

A significant amount of software research for developing programming environments for distributed memory multiprocessors is currently underway both in academia as well as industry. The research effort can be broadly categorized into three classes, namely parallelizing compilers, languages, and support tools. Recently, a standard set of extensions for Fortran 90, called High Performance Fortran (HPF) [8], has been developed which would provide a portable interface to a wide variety of parallel architectures.

The ESPRIT-3 project Prepare ([16]) aims to develop a High Performance Fortran compiler within an integrated programming environment.

This paper proposes techniques for processing HPF data distribution and data alignment specifications, array statements and procedure interfaces in the Prepare HPF Compiler in the basic stage which deals with the subset HPF. In the next section we describe the model underlying the compiler, and introduce the basic terminology used in the remaining sections. Section 3 introduces techniques for the basic restructuring of array statements. Processing procedure calls is discussed in Section 4 . The rest of the paper briefly deals with compiler optimizations (Section 5), and an overview of related work (Section 6), followed by the conclusion. The complete compiler specification can be found in [2].

\footnotetext{
* The work described in this paper was carried out as part of the ESPRIT research project $P$ 6516 PREPARE, and funded by the Austrian Research Foundation (FWF) and the Austrian Ministry for Science and Research (BMWF).
} 


\section{Basic Model and Terminology}

The parallelization process as described in this paper is based on the so-called $S P M D$ (Single Program Multiple Data) [11], or data parallel model of computation. Starting from the user specified data alignment and data distribution directives the compiler has to determine the layout of the data on the set virtual of processors and to determine how to spread the work among the processors available. Work distribution is achieved by applying the owner computes rule, so that each processor only computes those data elements that are allocated in its local memory. Access to non-local data is handled via explicit message passing.

\subsection{Basic Concepts}

In the following we assume that all declarations of objects (i.e. arrays, processor arrays, templates) are normalized such that the lower bound in each dimension is 1 . Figure 1 summarizes the notation used in this paper.

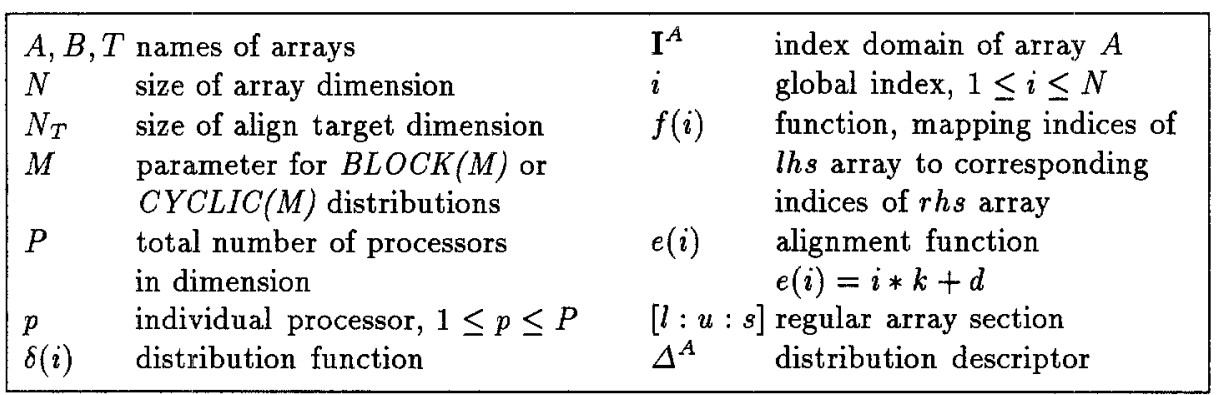

Fig. 1. Table of symbols used in this paper.

2.1.1 Processors. The set of processors, $W$ is represented in a program by one or more processor arrays. Any two processors in $W$ communicate by exchanging messages. Our model abstracts from the machine topology.

2.1.2 Data Distribution. A distribution of an array is defined by three components: the array index domain, the processor array index domain, and a distribution function. An index domain of rank $n$, denoted by $\mathbf{I}$, can be represented in the form $\mathrm{I}=\mathbf{X}_{i=1}^{n} D_{i}$, where the so-called index dimension $D_{i}$ is a non-empty, linearly order set of consecutive integers and $\mathbf{X}$ denotes the cartesian product.

Let in the following $\mathbf{I}_{i}^{A}$ and $\mathbf{J}_{j}^{R}$ denote the $i$-th index dimension of an array $A$ and $j$-th index dimension of a processor array $R$, respectively. A dimensional distribution function of dimension $i$ of $A$ to dimension $j$ of $R$ can be described by a total function from the array index domain $\mathbf{I}_{i}^{A}$ to the processors index domain $\mathbf{J}_{j}^{R}$, denoted by $\delta_{R_{j}}^{A_{i}}: \mathbf{I}_{i}^{A} \rightarrow \mathcal{P}\left(\mathbf{J}_{j}^{R}\right)-\phi$, where $\mathcal{P}\left(\mathbf{J}_{j}^{R}\right)$ denotes the power set of $\mathbf{J}_{j}^{R}$. 


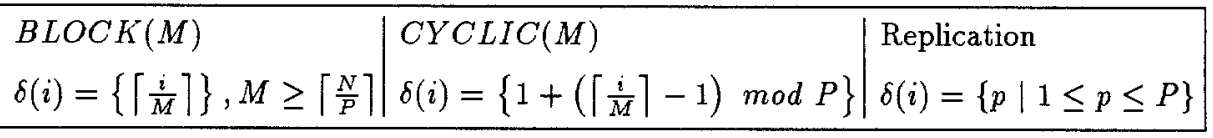

Fig. 2. HPF Distribution Functions.

The distribution functions provided by HPF are $B L O C K(M), C Y C L I C(M)$ and replication which is denoted by a '*'. They are summarized in Figure 2. A distribution for a multi-dimensional array is specified by describing the distribution of each array dimension separately, without any interaction of dimensions.

2.1.3 Alignment. Let $A$ denote a $n$-dimensional alignee and let $T$ denote a $m$-dimensional alignment target. An alignment for dimension $i$ of alignee $A$ with respect to dimension $j$ of the alignment target (or template) $T$ is defined by a so-called dimensional alignment function which is a total function $\alpha_{T_{j}}^{A_{i}}: \mathbf{I}_{i}^{A} \rightarrow \mathcal{P}\left(\mathbf{J}_{j}^{T}\right)-\phi$. The distribution function for alignee $A$ with respect to target $T$ can be constructed from the dimensional distribution functions.

\subsection{Data Layout}

The set of local elements of a distributed array $A$ (or template) on a particular processor $p$, denoted by local ${ }^{A}(p)$ contains all those elements of $A$ that are owned by $p$ and therefore are stored in $p$ 's local memory.

$$
\operatorname{local}^{A}(p)=\left\{i \mid \delta(i)=p \wedge i \in \mathbf{I}^{A}\right\}
$$

The local element set of an array is the basis for the computation of execution sets and communication sets and is also needed for the transformation of array declarations. The set of local template elements is needed for the computation of the local element sets for arrays that are aligned with that template. In the following local element sets are specified via closed form expressions, analog to Fortran 90 section notation.

2.2.1 Local Elements of a Distributee. Due to the nature of the HPF distributions $(B L O C K(M)$ and $C Y C L I C(M))$, the local element set of an array (template) dimension can be represented as a section by means of triplet notation. For $B L O C K, C Y C L I C(1)$ and $B L O C K(M)$ distributed dimensions the local element set can be described by a single triplet. This, however, might be not the case for $C Y C L I C(M)$ distributions, where the local element set of a processor may consist of more than one triplet, which complicates the handling of these sets at compile- and/or at runtime. The set of elements of a distributee $A$ owned by a particular processor $p$ (in global indices) is given by local ${ }^{A}(p)$ as defined in Figure 3.

In the case of a $C Y C L I C(M)$ distribution with $M<\left\lceil\frac{N}{P}\right\rceil$ some processors will get more than one section of array elements. The total number of sections on a particular processor $p$ is given by $n_{p}$. 


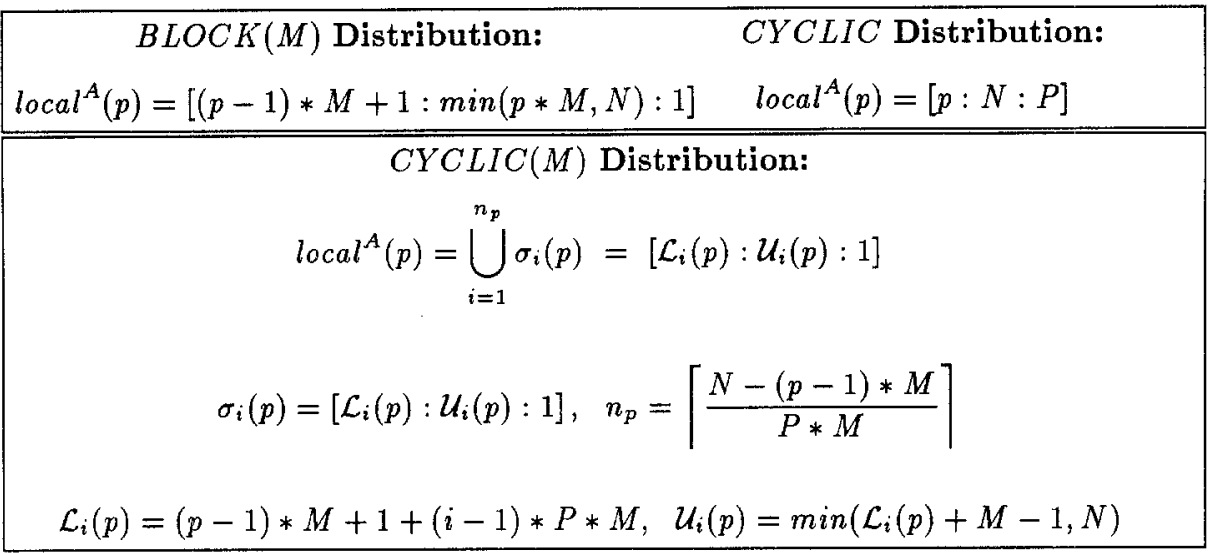

Fig. 3. Local Elements of a Distributee A.

2.2.2 Local Elements of an Alignee. The alignment functions included in subset HPF are restricted to linear functions of the alignment dummy. We assume that immediate alignment functions have been normalized into ultimate aligniment functions. The set of local elements on processor $p$ of an array $A(1$ : $N$ ), denoted by $\operatorname{local}_{T}^{A}(p)$, which is aligned with an alignment target $T$ by the alignment function $e(I)=k * I+d$ is determined by:

$$
\operatorname{local}_{T}^{A}(p)=e^{-1}\left(\text { local }^{T}(p) \cap e([1: N])\right)=e^{-1}\left(\text { local }^{T}(p)\right) \cap[1: N]
$$

The resulting formulas are presented in Figure 4. Note that for $\operatorname{BLOCK}(M)$ distributions $n_{p}$, the total number of sections, is always 1 .

$$
\operatorname{local} A_{T}^{A}(p)=\bigcup_{i=1}^{n_{p}} \sigma_{i}=\bigcup_{i=1}^{n_{p}}\left(\left[\mathcal{L}_{i T}^{A}(p): \mathcal{U}_{i T}^{A}(p): 1\right]\right)
$$

For $k>0$,

$$
\mathcal{L}_{i T}^{A}(p)=\max \left(1,\left\lceil\frac{\mathcal{L}_{i}^{T}(p)-d}{k}\right\rceil\right), \mathcal{U}_{i T}^{A}(p)=\min \left(N,\left\lfloor\frac{\mathcal{U}_{i}^{T}(p)-d}{k}\right\rfloor\right)
$$

and for $k<0$,

$$
\mathcal{L}_{i T}^{A}(p)=\max \left(1,\left\lceil\frac{\mathcal{U}_{i}^{T}(p)-d}{k}\right\rceil\right), \mathcal{U}_{i T}^{A}(p)=\min \left(N,\left\lfloor\frac{\mathcal{L}_{i}^{T}(p)-d}{k}\right\rfloor\right)
$$

where, $\mathcal{L}_{i}^{T}(p)$ and $\mathcal{U}_{i}^{T}(p)$ denote the lower and upper bound of local $l_{i}^{T}(p)$.

Fig. 4. Local Elements of an Alignee.

\subsection{Index Conversion}

In this section we define functions which convert global indices to local indices. 
$B L O C K(M)$ Distribution

$C Y C L I C(M)$ Distribution

$g 2 l(i)=1+(i-1) \bmod M$

$p=\left\lceil\frac{i}{M}\right\rceil, 1 \leq i \leq N, M \geq\left\lceil\frac{N}{P}\right\rceil$

$$
\begin{aligned}
& g 2 l(i)=(i-1) \bmod M+M *\left\lfloor\frac{i-1}{M * P}\right\rfloor+1 \\
& p=1+\left(\left\lceil\frac{i}{M}\right\rceil-1\right) \bmod P, 1 \leq i \leq N
\end{aligned}
$$

Fig. 5. Index Conversion for Distributees.

2.3.1 Index Conversion for Distributees. Given an array $A(1: N)$ with a $B L O C K(M)$ or $C Y C L I C(M)$ distribution onto $P$ processors, $1 \leq p \leq P$, and a global index $i$ of $A$, then the corresponding local index $l$ on processor $p$ is determined as shown in Figure 5.

\section{$B \operatorname{LOCK}(M)$}

$g 2 l(i)=i-\mathcal{L}_{T}^{A}(p)+1$

$p=\left\lceil\frac{i * k+d}{M}\right\rceil$

where $\mathcal{L}_{T}^{A}(p)$ is the lower bound of local $l_{T}^{A}(p)$ as in Figure 3.

$C Y C L I C(M)$

$g 2 l(i)=\left\lfloor\frac{n(i)-s_{p}}{a b s(k)}\right\rfloor * \Sigma_{p}+S P_{p}\left[\left(n(i)-s_{p}\right) \bmod a b s(k)\right]+o(i)-o_{p}+1$

$p=1+\left\lfloor\frac{i * k+d-1}{M}\right\rfloor \bmod P$

with

$n(i) \quad \ldots$ section number of index $i, i \in \sigma_{n(i)}(p)$

$o(i) \quad \ldots$ the offset of index $i$ in section $\sigma_{n(i)}(p)$

$s_{p} \quad \ldots$ the number of the first non-empty section, $\left|\sigma_{s_{p}}(p)\right|>0 \wedge\left|\sigma_{j}(p)\right|=0,1 \leq j \leq s_{p}-1$

$o_{p} \quad \ldots$ if $k>0$ : the offset of the first element in section $\sigma_{s_{p}}(p)$

if $k<0$ : the offset of the last element in section $\sigma_{s_{p}}(p)$

$S P_{p}\left[0: t_{p}\right] \ldots$ the so-called section-period table, with

$t_{p}=\min \left(n_{p}-s_{p}+1, a b s(k)\right)$

$S P_{p}[0]=0, S P_{p}[1]=o_{p}+\left|\sigma_{s p}\right|$,

$S P_{p}[j]=S P_{p}[j-1]+\left|\sigma_{s_{p}+j}\right|, 2 \leq j \leq t_{p}$

where $\Sigma_{p}=S P_{p}\left[t_{p}\right], n(i)=\left\lfloor\frac{i * k+d-1}{M * P}\right\rfloor+1, o(i)=\left\lfloor\frac{(i * k+d-1) \bmod M}{k}\right\rfloor$, and $n_{p}$ as in Figure 3 .

Fig. 6. Index Conversion for Alignees.

2.3.2 Index Conversion for Alignees. Let $A$ denote an array that is ultimately aligned with an alignment target $T$ by means of the alignment function $\epsilon(i)=i * k+d$. Let $T$ be distributed by $B L O C K(M)$ over $P$ processors. Given a global index $i$ of $A$ the corresponding local index $l$ on processor $p, 1 \leq p \leq P$ 
can be determined as shown in the left part of Figure 6 . For alignees with a $C Y C L I C(M)$ distributed target the index conversion is more complex.

\begin{tabular}{|c|c|c|c|}
\hline \multicolumn{2}{|l|}{ Layout of $\mathrm{T}$} & \multicolumn{2}{|l|}{ Layout of A } \\
\hline Processor 1 & Processor 2 & Processor 1 & Processor 2 \\
\hline 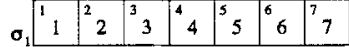 & \begin{tabular}{|l|l|l|l|l|l|l|l|l|}
$\sigma_{1}{ }^{1} 8$ & ${ }^{2}$ & 9 & 10 & 4 & 4 & 5 & 6 & 6 \\
\end{tabular} & $\sigma_{1}$ & 1 \\
\hline 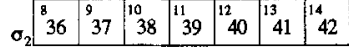 & 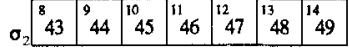 & $\left.\sigma_{2} 10\right|^{2} 11$ & $\sigma_{2}{ }^{3} 12{ }^{4} 13$ \\
\hline 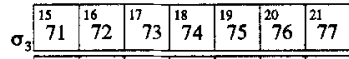 & \begin{tabular}{|c|c|c|c|c|c|c|c|}
$\sigma_{3}{ }_{3}^{15}$ & 16 & 79 & 17 & 18 & 19 & 20 & 21 \\
\end{tabular} & $\sigma_{3}$\begin{tabular}{|l|l|l|}
3 & 4 & 4 \\
\end{tabular} & 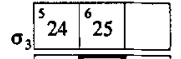 \\
\hline 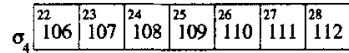 & 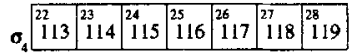 & \begin{tabular}{ll|l|} 
& 6 \\
& 33 & 734 \\
\end{tabular} & \begin{tabular}{|l|l|} 
& $0_{4}$ \\
\end{tabular} \\
\hline 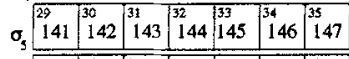 & 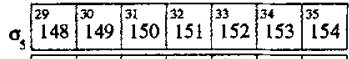 & $\sigma_{5}{ }_{45}^{8} 46$ & $\sigma_{5}$\begin{tabular}{|l|l|l|}
10 & 47 & 48 \\
\end{tabular} \\
\hline 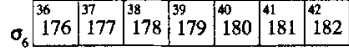 & 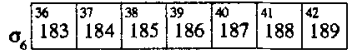 & 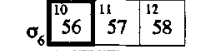 & $\sigma_{6}$\begin{tabular}{|l|l|l|}
12 & 13 & 13 \\
\end{tabular} \\
\hline 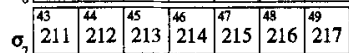 & 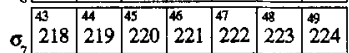 & $\sigma_{7}$\begin{tabular}{|l|l|l|}
13 & 14 \\
68 & 69 \\
\end{tabular} & 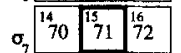 \\
\hline 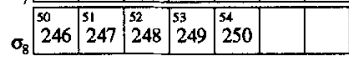 & & 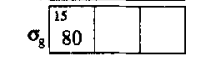 & \\
\hline & & $s_{1}-2$ & $s_{2}=1$ \\
\hline $\begin{array}{l}\text { member } \\
\text { of } A(1:: 5)\end{array}$ & & $o_{1}=0$ & $o_{2}=1$ \\
\hline globel index & & $S P_{1}=[0,2,5,7]$ & $\mathrm{SP}_{2}=[0,3,5,7]$ \\
\hline
\end{tabular}

REAL A (80), T (250)

!HPF\$ PROCESSORS R(5)

! HPF\$ ALIGN A(I) WITH T $(3 * I+8)$

!HPF $\$$ DISTRIBUTE $\mathrm{T}(\mathrm{CYCLIC}(7))$ ONTO $\mathrm{R}$

$A(1:: 5)=0$

Fig. 7. Layout of an Alignee with $C Y C L I C(M)$ Distributed Target

The set of local elements (in global indices) can be described by a union of sections $\sigma_{i}(p)$ as shown in Figure 4. However, depending on the alignment parameters, some sections at the beginning or at the end may be empty. In the following the number of the first non-empty section on a particular processor $p$ is denoted by $s_{p}$. The number of elements in each section may differ at most by 1 (except empty sections, the first non-empty section and the last nonempty section), with the maximum number of elements per section given by $\left\lceil\frac{M}{k}\right\rceil$ and the minimum number of elements per section given by $\left\lfloor\frac{M}{k}\right\rfloor$. The size of consecutive sections varies with a cycle of period length $k+1$. The fact that the length of the individual sections on a particular processor differ, complicates local address calculation. An additional problem is that the first sections might be smaller than $\left\lfloor\frac{M}{k}\right\rfloor$. We, therefore take the approach to determine the period of section lengthes, and the number of the first non-empty section on each processor, and incorporate this information in the formula for global to local index conversion which is depicted in Figure 6. The $S P_{p}$ table and the values for $\Sigma_{p}, s_{p}$ and $o_{p}$ 
have to be determined for each processor once and can be used for local address generation as well as for the representation of local element sets and the parallelization of array assignment statements.

In Figure 7 the layout of the arrays $A, T$ of the shown HPF code fragment and the corresponding $S P_{p}$ tables are visualized for the first two processors.

\subsection{Work Distribution}

As already mentioned, work distribution is derived on basis of the owner computes paradigm. The set of elements of the $l h s$ variable which have to be computed on a particular processor $p$ is referred to as execution set, denoted by exec $(p)$. If the lhs variable of the assignment statement is a regular array section, the execution set can be represented using Fortran 90 triplet notation.

Let $f$ denote the global index of the first element of the execution set on processor $p$, with $\operatorname{exec}(p) \neq \phi$. Let $A(p) \subseteq \operatorname{local}_{T}^{A}(p)$ denote the set of $\epsilon$ consecutive elements of $\operatorname{local}_{T}^{A}(p)$ starting from element with index $f$.

$$
\Lambda(p) \subseteq \operatorname{local}_{T}^{A}(p) . \quad|A(p)|=\epsilon, \quad \epsilon=S P\left[\frac{k}{\operatorname{gcd}(P, k)}\right] *\left\lceil\frac{s}{\operatorname{gcd}(P, s)}\right\rceil
$$

Let $\Xi(p)$ denote the set of the first $\xi$ elements $(1 \leq \xi \leq M)$ of the execution set on processor $p$ :

$$
\Xi(p)=\left\{x_{i} \mid x_{i} \in(\Lambda(p) \cap[l: u: s]), 1 \leq i \leq \xi\right\} .
$$

The execution set on processor $p$ in global indices can then be represented as follows:

$$
\operatorname{exec}(p)=\bigcup_{i=1}^{\xi}\left[x_{i}: u_{i}: \frac{s * M * P}{\operatorname{gcd}(P, k) * \operatorname{gcd}(P, s)}\right]
$$

By using the function $g 2 l(), \Sigma_{p}$ and the $S P$ table from Figure 6 the execution set in local indices is given by:

$$
\operatorname{exec\_ l}(p)=\bigcup_{i=1}^{\xi}\left[g 2 l\left(x_{i}\right): g 2 l\left(u_{i}\right): \frac{s * \Sigma_{p}}{g c d(P, k) * g c d(P, s)}\right]
$$

Fig. 8. Execution Sets for Array Assignments.

Let for an array assignment statement of the form $A(l: u: s)=\ldots, \mathbf{L}=$ $[l: u: s]$ denote the so-called $l h s$ reference space of array $A$. The execution set according to a an assignment to a regular array section $A(l: u: s)$ on a particular processor $p$ can be determined by the intersection of the $l h s$ reference space $\mathbf{L}$ with the local element set on this processor.

$$
\operatorname{exec}_{\mathbf{L}}^{A}(p)=\operatorname{local}^{A}(p) \cap[l: u: s]
$$

For $B L O C K(M)$ distributions the execution set can always be described via a single section whereas for $C Y C L I C(M)$ distributions this might not be the case. The summary of closed form expressions for exec(p) can be found in [2]. 
In the following we discuss in more detail the execution set generation for sections of alignees with a $C Y C L I C(M)$ distributed target. As it will be seen the execution set corresponding to an assignment to a regular array section $A(l: u: s)$ can be represented by means of at most $M$ array sections. To generate the execution set for an array section it is not necessary to determine the whole set $\operatorname{exec}(p)$, but it suffices to compute at most the first $M$ elements of $\operatorname{exec}(p)$, denoted by $\Xi(p)$. Note that for the computation of $\Xi(p)$ the intersection of the reference space $(l: u: s)$ with at most $k * s+1$ sections $\sigma_{i}(p), s_{p} \leq i \leq s_{p}+k * s$, has to be determined. In Figure 8 this method for the generation of execution sets for regular array sections is summarized. As can be seen from Figure 7 the execution set for the array section $A(1:: 5)$ for processor 1 is given in global

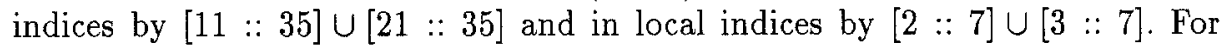
processor 2 the execution set consists of only 1 section: in global indices [1::35] and in local indices $[1:: 7]$.

\subsection{Communication Sets}

For the parallelization of assignment statements that reference distributed arrays, each processor has to determine the set of non-local elements it must receive from other processors in order to perform all the computations defined by its execution set. Furthermore it also has to determine which elements of its local element set have to be sent to other processors. Communication sets determine those elements a particular processor $p$ must send (or receive) to (from) another processor $q$, in order to compute the elements of its execution set.

Consider an assignment statement of the form $A\left(l_{1}: u_{1}: s_{1}\right)=B\left(\tilde{l}_{1}:\right.$ $\left.\tilde{u_{1}}: \tilde{s_{1}}\right)$. The set of elements a processor $p$ must send to a processor $q$ is called send_set $(p, q)$. It comprises those elements of $B$ that are in $p$ 's local memory and that are needed by another processor $q$. The set of non-local elements a processor $p$ needs from a processor $q$ is called recv_set $(p, q)$. Note that $\operatorname{recv} \_$set $(p, q)$ is given by $\operatorname{send} \_$set $(q, p)$

for each processor $q, q \neq p$

1. generate the execution set for processor $q$, $\operatorname{exec}_{\mathrm{L}}^{A}(q)$

2. determine $f\left(\operatorname{exec}_{\mathrm{L}}^{A}(q)\right)$, where $f(i)=\frac{\left(i-l_{1}\right)}{s_{1}} * \tilde{s_{1}}+\tilde{l_{1}}$

3. compute the intersection $f\left(\operatorname{exec}_{\mathrm{L}}^{A}(q)\right) \cap \operatorname{local}^{B}(p)$ which corresponds to send_set $^{B}(p, q)$

where $\mathbf{L}=\left[l_{1}: u_{1}: s_{1}\right]$.

Fig. 9. Communication Sets for Array Assignments.

In the case of array assignment statements computation of communication sets is based on regular section intersection. Computation of communication sets for an array assignment statement of the form shown above is summarized in Figure 9. 


\section{Processing Array Statements}

There are two main steps in the initial parallelization of an HPF program:

(1) Masking. Executable statements are masked (guarded) to ensure that all variable updates are exclusively in the local memory of the executing processor.

(2) Communication Insertion. Possible data movements between processors are specified by means of so-called communication descriptors COMMs which are generated and inserted into the program instead of concrete communication statements to enable later optimizations.

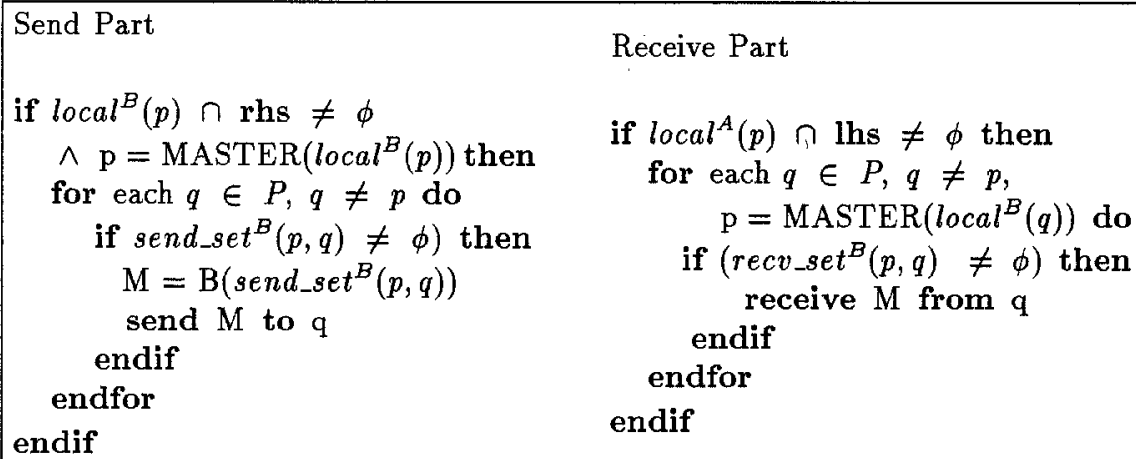

Fig. 10. Specification of the COMM Descriptor.

\subsection{High Level Communication Descriptor COMM}

A communication descriptor contains all information that is needed for the calculation of execution sets and communication sets using the formulas derived in Sections 2.4 and 2.5. Communication descriptors are flexible enough to allow for: recognizing and removing redundant communication descriptors, movement of descriptors (i.e. extraction from loops, movement across procedure boundaries), fusion of communication descriptors, splitting into the sending and receiving components. Moreover, there enable a uniform treatment of references to scalars, array elements, and array sections. So optimization of communication is performed entirely on the level of communication descriptors. In the final phase which adapts the SPMD program for the target machine, communication descriptors are transformed into the explicit message passing form.

Let lhs, rhs denote section subscripts and let $\Delta^{A}, \Delta^{B}$ denote distribution descriptors of arrays $A$ and $B$, respectively (each distributed array declared is represented by a descriptor). Let $\mathrm{M}$ denote a temporary buffer, and let $P$ denote the set of available processors, with arbitrary processors $p, q \in P$. An array communication descriptor of the form $\operatorname{COMM}\left(\Delta^{A},<\right.$ lhs $>, \Delta^{B},<$ rhs $>$, M), which for example is generated in case of an array assignment statement $A(\mathbf{l h s})=B$ (rhs), has the semantics introduced in the left part of Figure 10 when evaluated on a 
particular processor $p$. In this figure, $\operatorname{MASTER}(s)$ is a function which returns a uniquely defined processor $m$ which ownes the array element set $s$. This processor is responsible for sending the data. The choice of $m$ is system dependent. In many cases the overlap area communication approach can be used. Then $M$ will denote the overlap area description and the COMM will have the same semantics as the EXSR statement specified, for example, in $[5,6,17]$.

\begin{tabular}{|c|c|}
\hline & Transformed code \\
\hline & s1: WHERE (Owned(A(lhs))) A(lhs) $=\mathrm{S}$ \\
\hline Original code & $\begin{array}{l}\left.\text { s2: } \operatorname{COMM}\left(\Delta^{A},<\mathrm{lhs}>, \Delta^{B},<i\right\rangle, \mathrm{tmp}\right) \\
\quad \text { ! local accesses to B }\end{array}$ \\
\hline & WHERE (Owned(A(lhs)) .AND. \\
\hline !HPF $\$$ distribute $\mathrm{A}\left(\delta^{A}\right)$ & Owned $(\mathrm{B}(i))) \mathrm{A}(\mathbf{l h s})=\mathrm{B}(i)$ \\
\hline !HPF $\$$ distribute $\mathrm{B}\left(\delta^{B}\right)$ & $\begin{array}{l}\text { ! non-local accesses to B } \\
\text { WHERE (Owned(A(lhs)).AND. }\end{array}$ \\
\hline s1: $A($ lhs $)=S$ & .NOT. Owned $(\mathrm{B}(i))) \mathrm{A}(\mathrm{lhs})=\mathrm{tmp}$ \\
\hline s2: $\mathrm{A}(\mathrm{lhs})=\mathrm{B}(i)$ & s3: $\operatorname{COMM}\left(\Delta^{A},<\operatorname{lhs}>, \Delta^{B},<\right.$ rhs $>$, tmp $\_$B $)$ \\
\hline & ! local accesses to B \\
\hline s3: $A($ lhs $)=B(\mathbf{r h s})$ & WHERE (Owned(A(lhs)) .AND. \\
\hline & Owned $(\mathrm{B}(\mathbf{r h s}))) \mathrm{A}(\mathbf{l h s})=\mathrm{B}(\mathrm{rhs})$ \\
\hline & ! non-local accesses to $\mathrm{B}$ \\
\hline & WHERE (Owned(A(lhs)) .AND. \\
\hline & \\
\hline
\end{tabular}

Fig. 11. Transformation of Array Assignment Statements.

\subsection{Transformation of Array Assignments}

The first step is masking which replaces each array assignment statement $S$ of the program by the associated masked statement

\section{WHERE ( $\operatorname{mask}(S)) S$}

where $\operatorname{mask}(S)$ is expressed by a high level intrinsic function Owned(ref) which is also used for masking scalar assignments. The ref denotes the rhs reference which may be scalar or array valued of type logical. If ref is scalar valued the function returns the value.TRUE. if the array element denoted by ref is owned by the processor which is calling this function. If ref is array valued the function result is a logical array with the same shape as ref. Potential movement of nonlocal data is expressed by inserting the high level communication descriptor for the reference to a distributed array in the rhs.

If the rhs contains a distributed array the execution set of the statement is split into the local part that uses only data that is local on the executing processor, and the non-local part which uses some non-local data stored in the 
communication buffer (see transformation of statements $s 2$ and $s 3$ in Figure 11). Splitting also enables the subsequent optimizing transformation to achieve overlapping communication and computation (see Section 5).

Communication descriptors contain all the information that is needed for the calculation of execution sets and communication sets using the formulas derived in Sections 2.4 and 2.5. All possible cases that may arise during basic parallelization of array assignment statements are shown in Figure 11.

If on the right hand side more than one distributed array appears, a communication descriptor has to be generated for each of those arrays. However, in this case the splitting of the array assignment statement into local and non-local parts is more complex.

\subsection{Masked Array Assignment}

The goal of basic transformations applied to WHERE statements ${ }^{2}$ is to avoid communication for arrays involved in the mask evaluation at the time when the assignment statement is executed. Therefore it is necessary to enforce that the mask is aligned with the array on the left hand side of the array assignment statement. This is achieved by generating a temporary mask array, which is aligned with the array on the left hand side. Prior to the WHERE statement an assignment to the temporary mask array is inserted.

\section{CALL Statements and Procedure Interfaces}

The SPMD paradigm is applied to procedures in an obvious way: a procedure call in the node program is executed by all processors allocated to the program. We assume that for every procedure the interface is explicit to the caller. Moreover, if the INHERIT directive is used the user provides an explicit descriptive or prescriptive distribution for the dummy argument in question.

Different transformation techniques are applied depending on whether the dummy arrays are of assumed shape or explicit shape. In the following sections this is described in greater detail.

\subsection{Argument Passing via Assumed Shape Arrays}

The actual argument has to be redistributed, if necessary, to match the corresponding dummy argument distributed. In this case argument passing can no longer be call by reference, and a copy-in/copy-out semantics must be adopted. In order to allow for later optimization based on communication descriptor movement, redistribution is performed by the caller before and after the call to the procedure.

A temporary array with the same size and shape as the actual array (section) is allocated with the desired distribution and the actual array (section) is

\footnotetext{
${ }^{2}$ We assume that WHERE constructs are transformed into a series of WHERE statements during the program normalization phase.
} 
assigned to that temporary. Since this assignment may result in communication a high level communication descriptor is generated. In the CALL statement the original actual argument is replaced by the temporary. After the CALL statement the temporary array has to be reassigned to the original array, provided the INTENT(IN) attribute has not been specified for the corresponding dummy argument. This again may cause insertion of a communication descriptor.

\subsection{Argument Passing via Explicit Shape Arrays}

Argument passing using explicit shape dummy arrays is more complex than via assumed shape arrays because the restriction that the rank and shape of actual and formal array must be the same is absent. Therefore we must be able to handle cases where an $n$-dimensional actual array is associated with an $m$-dimensional formal array. Explicit shape dummies imply contiguous storage and rely on storage association between actual and formal array. Because the actual argument can be an array section we are forced to adopt in the basic parallelization phase the copy-in/copy-out argument transfer mechanism.

Original code

REAL A $\left(N_{1}, \ldots, N_{n}\right)$

!HPF\$ distribute $\delta^{A}:: \mathrm{A}$

CALL $\operatorname{Sub}(\mathrm{A}(\mathrm{sec}), \ldots)$ $\cdots$

\section{CONTAINS \\ SUBROUTINE $\operatorname{Sub}(X, \ldots)$ \\ REAL X $\left(M_{1}, \ldots, M_{m}\right)$ !HPF\$ distribute $\mathrm{X}\left(\delta^{X}\right)$ \\ END SUBROUTINE Sub}

END PROGRAM
Transformed code

REAL $\mathrm{A}\left(N_{1}, \ldots, N_{n}\right)$

!HPF $\$$ distribute $\delta^{A}:: \mathrm{A}$

REAL, ALLOCATABLE :: $T\left(: 1, \ldots,:_{m}\right)$ ...

! allocate $\mathrm{T}$ with distribution $\delta^{X}$,

ALLOCATE $\left(\mathrm{T}\left(M_{1}, \ldots, M_{m}\right)\right)$

$\mathrm{T}=\operatorname{RESHAPE}(\mathrm{A}, \operatorname{SHAPE}(\mathrm{T}))$

CALL Sub(T, ...)

$\mathrm{A}(\mathrm{sec})=$ RESHAPE $(\mathrm{T}, \operatorname{SHAPE}(\mathrm{A}(\mathrm{sec})))$

DEALLOCATE $(T)$

\section{CONTAINS}

! procedure interface stays unchanged

END PROGRAM

Fig. 12. Explicit Shape Dummy Arrays with Explicit Distribution.

Generally, if an array section is passed to an explicit shape dummy the array section has to be copied into a contiguous temporary array (Figure 12). This temporary is then passed to the procedure and assigned back to the original array section on exit form the procedure if necessary. If the distribution of a dummy argument array is specified explicitly, then the actual argument has to 
be redistributed, if necessary, to match it. Redistribution is achieved by allocating and assigning a temporary with the desired distribution. However, size and shape of this temporary are now determined by the dummy array rather than by the transferred section. Before each CALL statement to such a procedure an assignment of the original argument to the temporary array has to be generated. For those cases where the rank of the actual and formal argument differ this assignment is realized using the Fortran 90 RESHAPE function. The call to this intrinsic is later transformed into the call to a runtime library procedure.

The CALL statement has to be adjusted accordingly, and after the CALL statement the temporary array has to be assigned back to the original argument, provided the INTENT(IN) attribute has not been specified.

\section{Optimizations}

In this phase, the compiler applies transformations to improve performance of the resulting SPMD program. The program analysis must capture sufficient information to enable sophisticated optimizations. Data flow analysis information, for example, enables to decide whether execution and communication sets computed for an array statement can be reused for some of the following ones.

Original code

$\mathrm{A}(\mathbf{l h s})=\mathcal{F}(\mathrm{B}(\mathbf{r h s})) ! \mathcal{F}$ denotes operations on elements of $\mathrm{B}$

Transformed code

$\operatorname{COMMS}\left(\Delta^{A},<\right.$ lhs $>, \Delta^{B},<$ rhs $>$, tmp_B $)$

! computations accessing only local elements of $B$ - this hides the latency

WHERE (Owned(A(lhs)) .AND. Owned(B(rhs))) A(lhs) $=\mathcal{F}(\mathrm{B}($ rhs $))$

$\operatorname{COMMR}\left(\Delta^{A},\left\langle\right.\right.$ lhs $>, \Delta^{B},<$ rhs $>$, tmp_B $)$

! computations accessing non-local elements of $B$

WHERE (Owned(A(lhs)) .AND..NOT.Owned(B(rhs))) A(lhs) $=\mathcal{F}($ tmp_B $)$

Fig. 13. Hiding latency by local computation.

There are several compiler optimizations which can reduce or hide the communication latency overhead. One of the approaches is called the latency tolerance [10]. The aim is to hide the message transmission time, by overlapping communication with parallel computation. The execution set for each processor is split into local and nonlocal parts in the way which was discussed in Section 3.2. Each processor executes separately parts of expressions which access only local data and those which access nonlocal data as expressed in Figure 13. COMM statements are split into send (COMMS) and receive (COMMR) parts 
whose semantics is clear from the specification introduced in Figure 10. COMMS is moved before the part performing local computations, and COMMR between this part and the one performing computations in which remote accesses are needed.

\section{Related Work}

In this paper we propose the systematic solution for general $B L O C K(M)$ and CYCLIC(M) distributions allowing arbitrary HPF alignment functions. Solutions of many special cases of the problems discussed in this paper appeared in the literature. Li and Chen specified interprocessor communication by the descriptor called communication pattern. They used this descriptor in communication optimization. Koelbel [12] treats special communication set generation cases for data-parallel loops where arrays are distributed using a $B L O C K$ or a $C Y C L I C$ distribution without considering any alignment aspects. Gupta et al. [7] extend Koelbel's techniques to $C Y C L I C(M)$ distributions and introduce a procedure for performing index conversion. Chatterjee at al. [4] also allow an arbitrary HPF alignment function. They construct a finite state machine to convert global indices of a regular array section to local ones. Unlike our approach, they do not preserve array sections after the index conversion. Moreover this kind of conversion cannot be applied to vector subscripted accesses. Stichnoth's approach [15] only allows alignment strides to be 0 or 1 . Compiler communication optimizations are discussed in $[9,13]$. Optimization techniques based on the runtime processing were developed by Saltz et al. [14], and the paper [3] describes their implementation in the SUPERB System.

\section{Concluding Remarks}

The techniques described in this paper are developed within the context of our research whose results have been documented in [2]. In the paper, we showed how data alignment and data distribution specifications are translated into mathematical functions that determine the ownership and adressing of local data. We introduced the methodology for derivation of execution sets applying the owner computes rule and derivation of communication sets. All the above terms create the basis for the implementation of masks and communication descriptors. The techniques described are now being implemented within the Prepare project and within the Vienna Fortran 90 ([1]) compiler.

\section{Acknowledgment}

The authors would like to thank the Prepare Project partners from ACE Amsterdam, TNO Delft and IRISA Rennes for useful discussions and comments on the document [2]. 


\section{References}

1. S.Benkner, B.Chapman, H.P.Zima. Vienna Fortran90. In Proceedings of the Scalable High Performance Computing Conference, Williamsburg, USA, pp. 51-59, April 1992.

2. S. Benkner, P. Brezany, H.P. Zima. Functional Specification of the Prepare Parallelization Engine. Internal Report of the Prepare Project, June 1993.

3. P.Brezany, M.Gerndt, V.Sipkova, H.P.Zima. SUPERB Support for Irregular Scientific Computations. In Proceedings of the Scalable High Performance Computing Conference, Williamsburg, USA, pp. 314-321, April 1992.

4. S. Chatterjee, J.R. Gilbert, F.J. Long, R. Schreiber, S-H. Teng. Generating Local Addresses and Communication Sets for Data-Parallel Programs. In Proceedings of the Fourth ACM SIGPLAN Symposium on Principles \& Practice of Parallel Programming (PPOPP), 149-158, San Diego, May 19-22, 1993.

5. H.M. Gerndt, Updating Distributed Variables in Local Computations, Concurrency: Practice and Experience, Vol. 2(3), 171-193 (September 1990)

6. H.M. Gerndt. Program Analysis and Transformations for Message-Passing Programs. Proceedings of the Scalable High Performance Computing Conference, Williamsburg, Virginia, 60-67, April 26-29, 1992

7. S. K. S. Gupta et al. Compiling Array Expressions for Efficient Execution on Distributed-Memory Machines. Proceedings of ICPP'93.

8. High Performance FORTRAN Forum. High Performance FORTRAN Language Specification, Version 1.0, Rice University, Houston, TX, May 1993.

9. S. Hiranandani, K. Kennedy, C. Tseng. Compiler Optimizations for Fortran $D$ on MIMD distributed-memory machines. In Proc. of Supercomputing'91, Albuquerque, NM, November 1991.

10. H. F. Jordan. Scalability of Data Transport. Proceedings of the Scalable High Performance Computing Conference, Williamsburg, Virginia, 1-8, April 26-29, 1992.

11. A.H. Karp. Programming for Parallelism. Computer 20(5), 43-57, May 1987

12. C. Koelbel. Compiling Programs for Nonshared Memory Machines. Ph.D. Dissertation, Purdue University, West Lafayette, IN, November 1990.

13. J. Li and M. Chen. Compiling Communication-Efficient Programs for Massively Parallel Machines. IEEE Transactions on Parallel and Distributed Systems, Vol.2(3), 361-376, July 1991.

14. J. Saltz, K. Crowley, R. Mirchandaney, and H. Berryman. Run-time scheduling and execution of loops on message passing machines. Journal of Parallel and Distributed Computing, 8(2):303-312, 1990.

15. J.M. Stichnoth. Efficient Compilation of Array Statements for Private Memory Multicomputers. Research Report CMU-CS-93-109, Carnegie Mellon University, Pittsburgh, February, 1993.

16. A. Veen, M. de Lange. Overview of the Prepare Project. In Proceedings of the Fourth Workshop on Compilers for Parallel Computers, Delft, Holland, December 1993.

17. H. Zima, B. Chapman. Compiling for Distributed-Memory Systems. Proc. of the IEEE, vol 81, n. 2, February 1993. 